\title{
Salutogenesis beyond Health
}

\author{
Shifra Sagy
}

Aaron Antonovsky was my mentor in the long journey of writing my doctoral dissertation, which was the first to be written using the salutogenic paradigm framework. He was not only my academic advisor but also had a tremendous impact on my life. For me, the salutogenic model is not only a theoretical paradigm, whose genesis I witnessed and later on took an active part in its development. For me, this theory is the basis for a meaningful understanding of my life story, a story that has been embedded in the conflictual Jewish existence in Israel. Aaron and his salutogenic ideas have guided me in this difficult path too.

Aaron Antonovsky enriched us with a unique, challenging model, which had high levels of comprehensibility, manageability, and especially meaningfulness. When he passed away 25 years ago, I wondered whether, and perhaps how, the model would be developed after him. To witness today, the rich and active development of the model is deeply exciting. Thus, I am truly enthusiastic about taking part in this endeavor-the second edition of The Handbook of Salutogenesis_and especially pleased to edit this part dealing with new developments and creative advancements of the theory.

It was very tempting to continue Antonovsky's way by using his strict guidelines for salutogenic research. However, Aaron also taught me that "it is wise to see models, theories, constructs, hypotheses, and even ideas as heuristic devices, not only truths" (Antonovsky, 1996, p. 246). The chapters included in this part of the handbook represent good examples of following this advice.

In Chap. 22, my colleague Adi Mana and I ask how to broaden the salutogenic paradigm's scope into an interdisciplinary framework and include other social concepts in its research. As one example of such interdisciplinary research, we review some new studies in conflict areas investigating intergroup relations. By relating

S. Sagy $(\bowtie)$

Martin Springer Center for Conflict Studies,

Ben-Gurion University of the Negev, Be'er Sheva, Israel

e-mail: shifra@bgu.ac.il to such areas of research, we try to address not only the "classic" question-who copes successfully and stays healthy-but also other salutogenic questions such as "who expresses more openness to the other?"

In the 2017 edition of the Handbook of Salutogenesis, I suggested that these meaningful questions, stemming from our political and social reality, should be extensively discussed in the framework of salutogenesis. Indeed, since the 2017 edition of the handbook, there has been impressive advancement in this research direction, including the development of new concepts like the sense of national coherence.

The 2020 worldwide outbreak of the COVID-19 pandemic has shaken up lives and transformed perceptions about "normality." As of this writing, the pandemic is stimulating salutogenic-oriented interdisciplinary health research and social research-this chapter reviews some cutting-edge COVID-19 social studies beyond health.

In Chap. 23, Stephen Joseph and I propose integrating two paradigms - positive psychology and salutogenesisand suggest a joint conceptual framework, which we term as "salutogenic positive psychology." Despite the differences between the two movements and their different theoretical roots, we believe that the integrative approach has greater utility in advancing psychological research on mental health and well-being.

The other three chapters in Part IV answer our call for interdisciplinary salutogenic-oriented research and discuss its possible application in three areas.

In Chap. 24, Maass, Kiland, Espnes, and Lillefjord thoroughly discuss the different possibilities of applying salutogenesis in politics and public policy. Politics is one of the upstream conditions that shape our individual lives as well as our society. Thus, asking about if and how salutogenesis can be applied to this field appears to be a most significant subject in Part IV of the handbook relating to salutogenesis beyond health. In all of his writing about his model, Antonovsky emphasized how politics and policies contribute to shaping individual and collective abilities to strengthen 
salutogenic resources. The authors of this chapter approach this issue from the opposite direction, asking, "how can salutogenesis contribute to outlining strategies and structural processes linked to politics and policy-making?" Their creative discussion succeeds in bringing the reader the utility of the salutogenic approach in addressing such issues.

In Chap. 25, Levasseur and Naud discuss some important aging factors that could increase the likelihood of a stronger SOC: aging at home, participation, and social support. In his last paper, Antonovsky (1996) highlighted an example of an intervention among older people, living in their homes, who refused to accept help. He suggested that if researchers had been guided by the salutogenic question of "how to strengthen the comprehensibility, manageability, and meaningfulness of elders," their intervention research could have been much more sophisticated and rich. Levasseur and Naud are addressing this call. In their chapter, they analyze how social support, active participation, mobility, and other factors can strengthen SOC in old age. They also bring some examples of individual and community programs that are already operating within this salutogenic orientation.

In Chap. 26, Golembiewski suggests adding another domain in our life to be viewed through the salutogenic lens: architectural design. In a creative and explorative discussion, the author analyzes detailed and concrete examples and offers ideas on how architecture can advance comprehensibility, manageability, and meaningfulness in our lives.

In Chap. 4 of this handbook, we wrote that Aaron taught us that the most meaningful advancement in scientific work is to ask good questions. I trust that our part of the handbook succeeds in relating to this challenge.

\section{Reference}

Antonovsky, A. (1996). The salutogenic model as a theory to guide health promotion. Health Promotion International, 11(1), 11-18.

Open Access This chapter is licensed under the terms of the Creative Commons Attribution 4.0 International License (http://creativecommons. $\mathrm{org} /$ licenses/by/4.0/), which permits use, sharing, adaptation, distribution and reproduction in any medium or format, as long as you give appropriate credit to the original author(s) and the source, provide a link to the Creative Commons license and indicate if changes were made.

The images or other third party material in this chapter are included in the chapter's Creative Commons license, unless indicated otherwise in a credit line to the material. If material is not included in the chapter's Creative Commons license and your intended use is not permitted by statutory regulation or exceeds the permitted use, you will need to obtain permission directly from the copyright holder. 